\title{
СРАВНЕНИЕ ВЛИЯНИЯ НЕЛИНЕЙНОСТЕЙ ПЕРВОГО РОДА И ДВОЙНОЙ НЕЛИНЕЙНОСТИ НА ТЕПЛОПРОВОДНОСТЬ ПЛАСТИН ИЗ СПЛАВОВ АЛЮМИНИЯ, ТИТАНА И СТАЛИ
}

\section{Е. П. Зайцев}

Летная академия национального авиационного университета

ул. Добровольского, 1, г. Кропивницкий, 25005, Украина. E-mail: zaytsev.ep.klanau @ gmail.com

На основе приближенного метода суперпозиции решения краевых задач дважды нелинейной нестационарной теплопроводности, рассмотрено влияние термочувствительности рассмотренных материалов и лучистоконвективного теплообмена на распределение температурных полей по толщине и времени, в центре и поверхности пластины. На большом количестве числовых расчетов, графически показаны качественные изменения влияния нелинейностей на теплопроводность пластин при различных значениях коэффициента теплоотдачи и температуры окружающей среды. Полученные в работе результаты могут быть использованы в инженерной практике в качестве тестовых решений при конструировании и расчёте режимов высокотемпературного теплообмена элементов конструкций, а также критерием оценки для соответствующих численных решений.

Ключевые слова: теплопроводность, суперпозиция, термочувствительность, лучисто-конвективный теплообмен, преобразования Гудмэна и Кирхгофа, ряды Фурье.

\section{ПОРІВНЯННЯ ВПЛИВУ НЕЛІНІЙНОСТІ ПЕРШОГО РОДУ І ПОДВІЙНОЇ НЕЛІНІЙНОСТІ НА ТЕПЛОПРОВІДНІСТЬ ПЛАСТИН ІЗ СПЛАВІВ АЛЮМІНІЮ, ТИТАНУ І СТАЛІ}

\section{Є. П. Зайцев}

Льотна академія національного авіаційного університету

вул. Добровольського, 1, м. Кропивницький, 25005, Україна. E-mail: zaytsev.ep.klanau @ gmail.com

На сучасному етапі розвитку техніки необхідно максимально забезпечити міцність, надійність і довговічність конструкційних матеріалів, успішно функціонуючих в умовах високотемпературного теплообміну. У зв'язку з цим, широке застосування знаходять отримані за останній час нові сплави алюмінію і титану, які застосовуються для деталей космічних і повітряних літальних апаратів, парових і газових турбін, реактивних і ракетних двигунів. Метою дослідження $є$ застосування нового підходу до вирішення задач теплопровідності для оцінки впливу нелінійностей першого роду і подвійної нелінійності на розподіл температурних полів в алюмінієвих і титанових сплавах, які мають суттєві відмінності коефіцієнтів теплопровідності. При вирішенні поставленої задачі, перетвореної до безрозмірного вигляду, застосовуються перетворення Гудмена і Кірхгофа, потім відносна температура і функції від неї розкладаються в ряд за синусами на апріорному проміжку, далі застосовується принцип суперпозиції, після чого вихідна постановка перетворюється до сукупності лінеаризованих задач. Лінійні задачі вирішуються методом інтегральних перетворень, після чого підсумовуються. Верхня межа апріорного проміжку визначається 3 умови, що відносна температура, отримана 3 вирішеної задачі при $F o \rightarrow \infty$, приймає значення верхньої межі апріорного проміжку. Розроблений аналітичний метод і отримання якісних і кількісних числових результатів для оцінки впливу нелінійностей на розподіл температурних полів в елементах конструкцій з алюмінієвих і титанових сплавів, дозволяє найбільш повно і адекватно враховувати властивості матеріалу і високотемпературний теплообмін 3 навколишнім середовищем. Отримані результати можуть бути використані в розрахунковій практиці теплотехнічних лабораторій для оцінки експериментальних досліджень матеріалів, що піддаються впливу високотемпературного теплообміну, а також критерієм оцінки відповідних числових рішень. Подальші дослідження будуть спрямовані на порівнянні впливу нелінійностей і анізотропії для ортотропной пластини в широкому діапазоні зміни коефіцієнта теплопровідності.

Ключові слова: теплопровідність, суперпозиція, термочутливість, конвективно-променевий теплообмін, перетворення Гудмена і Кірхгофа, ряди Фур'є.

АКТУАЛЬНОСТЬ РАБОТЫ. На современном этапе развития техники необходимо максимально обеспечить прочность, надежность и долговечность конструкционных материалов, успешно функционирующих в условиях высокотемпературного теплообмена (терморадиационного, аэродинамического, комбинированного лучисто-конвективного и др.). В связи с этим, широкое применение находят полученные за последнее время новые сплавы алюминия и титана, которые применяются для деталей космических и воздушных летательных аппаратов, паровых и газовых турбин, реактивных и ракетных двигателей. Возникающие при этом большие градиенты температуры требуют в соответствующей математической постановке задачи теплопроводности учета, как зависимостей теплофизических характеристик материала от температуры, так и высокотемпе- ратурного теплообмена с окружающей средой, что делает поставленную задачу дважды нелинейной [1]. Это позволяет получать более точные решения, удобные для анализа теплового режима твердого тела, выделять превалирующие факторы теплообмена и использовать полученные результаты для последующего анализа термонапряженного состояния тела. На пути решения этой проблемы существенного значения приобретают вопросы более точного определения и оценки влияния нелинейностей в алюминиевых [2] и титановых сплавах, которые имеют значительные различия коэффициентов теплопроводности. В работе, для более наглядной графической картины оценки такого влияния, в качестве промежуточных числовых расчетов, использовалась так же низкоуглеродистая сталь. 
При решении задач нестационарной теплопроводности, различные типы нелинейностей учитывались в работах [3-10], однако, среди них незначительная часть работ решалась в дважды нелинейной постановке, с последующим численным анализом влияния нелинейностей на распределение относительной температуры.

Важность и актуальность решаемой задачи состоит в том, что разработка приближенного аналитического метода и получение качественных и количественных числовых результатов для оценки влияния нелинейностей на распределение температурных полей в элементах конструкций из алюминиевых и титановых сплавов, позволяет наиболее полно и адекватно учитывать свойства материала и высокотемпературный теплообмен с окружающей средой. Это позволит использовать полученные результаты в расчетной практике теплотехнических лабораторий для оценки экспериментальных исследований материалов, подверженных воздействию высокотемпературного теплообмена, а также критерием оценки соответствующих числовых решений.

Целью работы является применение метода суперпозиции [1], на качественном сравнении влияния нелинейностей первого рода и двойной нелинейности, на распределение температурных полей в элементах конструкций из алюминиевых и титановых сплавов, которые имеют существенные различия коэффициентов теплопроводности, что позволит использовать полученные результаты в расчетной практике теплотехнических лабораторий.

МАТЕРИАЛ И РЕЗУЛЬТАТЫ ИССЛЕДОВАНИЙ. Рассмотрим неограниченную пластину толщиной $2 R$. Начальное распределение температуры постоянно и одинаково $T(x, 0)=T_{0}$. В начальный момент времени пластина помещается в среду с постоянной температурой $T_{\tilde{n}}>T(x, 0)$. Между ограничивающими поверхностями пластины и окружающей средой происходит лучисто-конвективный теплообмен. Необходимо найти распределение температуры по времени и толщине для нелинейностей 1-го рода и двойной нелинейности, а также их влияние на температурное поле пластины по сравнению с линейным решением для сплавов алюминия, титана и низкоуглеродистой стали.

Нестационарное температурное поле в пластине может быть определено из решения следующей краевой задачи, записанной в размерном виде:

$$
\begin{aligned}
\circ & C_{v}(T) T_{, t}=\left(\lambda(T) T_{, x}\right)_{, x},(t>0 ; \quad-R<x<R) ; \\
& \left.T(x, t)\right|_{t=0}=0 ;\left.\quad T_{, x}\right|_{x=0}=0 ;\left.\lambda(T) T_{, x}\right|_{x=R}= \\
= & \left.\left\{\alpha\left(T_{c}-T(R, t)\right)+\sigma_{\hat{\mathrm{a}}}\left(T_{\tilde{n}}^{4}-T^{4}(R, t)\right)\right\}\right|_{x=R}, \bullet
\end{aligned}
$$

где $\bigcirc$ и • - начало и конец группы соотношений; $t$-время; $x$ - текущая координата по толщине пластины; $T$-абсолютная температура тела; $T_{0}$-начальная температура тела; $T_{c}$ - температура среды;
$\lambda(T)$ - коэффициент теплопроводности изотропного тела; $C_{v}(T)$ - объемная теплоемкость; $\sigma_{\hat{\mathrm{a}}}=\varepsilon \sigma_{0}-$ приведенный коэффициент теплообмена излучением; $\varepsilon$-степень черноты; $\sigma_{0}$ - постоянная СтефанаБольцмана; $\alpha$-коэффициент теплоотдачи.

Преобразуем поставленную задачу (1) к следующему безразмерному виду относительно $\theta(X, F o)=\left(T(x, t)-T_{0}\right) / T_{z}:$

$$
\begin{gathered}
\circ C_{v}^{*}(\theta) \theta_{, F \mathrm{o}}=\left(\lambda_{x}^{*}(\theta) \theta_{, X}\right)_{, X} \quad(0<\rho<1, \quad F o>0) ; \\
\left.\theta_{, X}\right|_{X=0}=0 ;\left.\quad \theta(X, F o)\right|_{F o=0}=0 ;\left.\quad \lambda^{*}(\theta) \theta_{, X}\right|_{X=1}= \\
=Q-\left.\left(G \theta+S k\left(\theta^{4}+4 \theta^{3} S+6 \theta^{2} S^{2}\right)\right)\right|_{X=1}, \bullet
\end{gathered}
$$

где теплофизические характеристики представлены в виде произведения двух множителей $\lambda(T)=\lambda_{0} \lambda^{*}(\theta), C_{v}(T)=C_{v 0} C_{v}^{*}(\theta)$ из которых первый с индексом «нуль» имеет размерность и не изменяется, а второй, обозначенный звездочкой, безразмерный и является функцией $\theta ; \quad X=x / R$; $a=\lambda_{0} / C_{v 0} ; F o=a t / R^{2} ; Q=B i+S k P ; \quad B i=\alpha R / \lambda_{0} ;$ $S k=\sigma_{\hat{\mathrm{a}}} R T_{\tilde{n}}^{3} / \lambda_{0} ; \quad G=B i+4 S k \cdot S^{3} ; \quad T_{z}=T_{-}-{ }_{0} T$ $S=T_{0} / T_{z} ; \quad P=\left(T_{c}^{4}-T_{0}^{4}\right) / T_{z}^{4}$. [1]

Вводя в (2) преобразования Гудмэна и Кирхгофа

$$
A(\theta)=\int_{0}^{\theta} C_{v}^{*}\left(\theta^{\prime}\right) d \theta^{\prime} \quad B(\theta)=\int_{0}^{\theta} \lambda^{*}\left(\theta^{\prime}\right) d \theta^{\prime}
$$

с помощью дифференциальных соотношений для перехода к новым функциям

$$
A(\theta)_{, F o}=C_{v}^{*}(\theta) \theta_{, F o}, B(\theta)_{, X}=\lambda^{*}(\theta) \theta_{, X},
$$

полученным из (3) при их дифференцировании по соответствующим координатам, систему (2) представим в следующей эквивалентной форме:

$$
\begin{gathered}
\circ A(\theta)_{, F o}=B(\theta)_{, X X} \\
\left.\theta(X, F o)\right|_{F o=0}=0 ;\left.\quad \theta_{, X}\right|_{X=0}=0 \\
\left\{B(\theta)_{, X}=Q-\left(G \theta+S k\left(\theta^{4}+4 \theta^{3} S+6 \theta^{2} S^{2}\right)\right)\right\}_{X=1}
\end{gathered}
$$

В (4) функции (3) и $\theta^{(m)}(m=\overline{1,4})$ непрерывны и удовлетворяют условиям Дирихле [11] на априорно выбранном интервале $\left(0, \theta_{P}\right)$ ( $\theta_{P}$-пока неопределенная постоянная), а, следовательно, их можно представить в виде следующих сходящихся рядов Фурье по синусам:

$$
\begin{gathered}
\theta=\sum_{k=1}^{\infty} \alpha_{k}^{(1)} \sin \left(k \pi \theta / \theta_{P}\right)=\sum_{k=1}^{\infty} \theta^{(k)} \\
\left(A(\theta), B(\theta), \theta^{(m)}\right)= \\
=\sum_{k=1}^{\infty}\left(a_{k} / \alpha_{k}^{(1)}, b_{k} / \alpha_{k}^{(1)}, \alpha_{k}^{(m)} / \alpha_{k}^{(1)}\right) \theta^{(k)},
\end{gathered}
$$


где

$$
\begin{gathered}
\left(a_{k}, b_{k}, \alpha_{k}^{(m)}\right)= \\
=\frac{2}{\theta_{P}} \int_{0}^{\theta_{P}}\left(A(\theta), B(\theta), \theta^{m}\right) \sin \frac{k \pi \theta}{\theta_{P}} d \theta, \\
\circ \alpha_{k}^{(1)}=\frac{2 \theta_{P}(-1)^{k+1}}{k \pi}, \quad \alpha_{k}^{(3)}=\frac{2 \theta_{P}^{3}(-1)^{k+1}\left[(k \pi)^{2}-6\right]}{(k \pi)^{3}}, \\
\alpha_{k}^{(2)}=2 \theta_{P}^{2}\left\{(-1)^{k+1}(k \pi)^{2}-2\left[(-1)^{k+1}+1\right]\right\} /(k \pi)^{3}, \\
\alpha_{k}^{(4)}=\frac{2 \theta_{P}^{4}\left\{(-1)^{k+1}\left[(k \pi)^{4}-12(k \pi)^{2}+24\right]+24\right\}}{(k \pi)^{5}} .
\end{gathered}
$$

С учетом (5), (6) и разложения экспоненты $e^{x}$ в ряд Маклорена при $x=1$, т. е. $1=\sum_{k=1}^{\infty} e^{-1} /(k-1) !$, (4) перепишем в виде:

$$
\begin{gathered}
\circ\left(\sum_{k=1}^{\infty}\left(a_{k} / \alpha_{k}^{(1)}\right) \theta^{(k)}\right)_{, F o}=\left(\sum_{k=1}^{\infty}\left(b_{k} / \alpha_{k}^{(1)}\right) \theta^{(k)}\right)_{, X X} ; \\
\left.\sum_{k=1}^{\infty} \theta^{(k)}\left(X, F O_{k}\right)\right|_{F o_{k}=0}=0 ;\left.\left(\sum_{k=1}^{\infty} \theta^{(k)}\right)_{, X}\right|_{X=0}=0 ; \\
\left(\left.\left.\sum_{k=1}^{\infty} \frac{b_{k}}{\alpha_{k}^{(1)}} \theta^{(k)}\right|_{, X}\right|_{X=1}=Q \sum_{k=1}^{\infty} \frac{1}{e(k-1) !}-\left\{G \sum_{k=1}^{\infty} \theta^{(k)}+\right.\right. \\
+S k \sum_{k=1}^{\infty} \frac{\alpha_{k}^{(4)}}{\alpha_{k}^{(1)}} \theta^{(k)}+4 S \sum_{k=1}^{\infty} \frac{\alpha_{k}^{(3)}}{\alpha_{k}^{(1)}} \theta^{(k)}+ \\
\left.\left.+6 S^{2} \sum_{k=1}^{\infty} \frac{\alpha_{k}^{(2)}}{\alpha_{k}^{(1)}} \theta^{(k)}\right)\right\}\left.\right|_{X=1} ^{\bullet}
\end{gathered}
$$

Внесем частные производные под знаки сумм, получим:

$$
\begin{gathered}
\circ \sum_{k=1}^{\infty} \frac{a_{k}}{\alpha_{k}^{(1)}} \frac{\partial \theta^{(k)}}{\partial F o}=\sum_{k=1}^{\infty} \frac{b_{k}}{\alpha_{k}^{(1)}} \frac{\partial^{2} \theta^{(k)}}{\partial X^{2}} ; \\
\left.\sum_{k=1}^{\infty} \theta^{(k)}\left(X, F O_{k}\right)\right|_{F o_{k}=0}=0 ;\left.\quad \sum_{k=1}^{\infty} \frac{\partial \theta^{(k)}}{\partial X}\right|_{X=0}=0 \\
\left.\sum_{k=1}^{\infty} \frac{b_{k}}{\alpha_{k}^{(1)}} \frac{\partial \theta^{(k)}}{\partial X}\right|_{X=1}=Q \sum_{k=1}^{\infty} \frac{1}{e(k-1) !}-\left\{G \sum_{k=1}^{\infty} \theta^{(k)}+\right. \\
+S k\left(\sum_{k=1}^{\infty} \frac{\alpha_{k}^{(4)}}{\alpha_{k}^{(1)}} \theta^{(k)}+4 S \sum_{k=1}^{\infty} \frac{\alpha_{k}^{(3)}}{\alpha_{k}^{(1)}} \theta^{(k)}+\right. \\
\left.\left.+6 S^{2} \sum_{k=1}^{\infty} \frac{\alpha_{k}^{(2)}}{\alpha_{k}^{(1)}} \theta^{(k)}\right)\right\}\left.\right|_{X=1} ^{\bullet}
\end{gathered}
$$

Применяя к (10) обобщенный принцип суперпозиции [3], получим совокупность линеаризованных задач теплопроводности относительно $\theta^{(k)}$ :

$$
\begin{gathered}
\circ \theta_{, F o_{k}}^{(k)}=\theta_{, X X}^{(k)} ;\left.\quad \theta^{(k)}\left(X, F o_{k}\right)\right|_{F o_{k}=0}=0 ;\left.\quad \theta_{, X}^{(k)}\right|_{X=0}=0 ; \\
\left.\left\{\theta_{, X}^{(k)}+B i_{k} \theta^{(k)}\right\}\right|_{X=1}=M_{k}, k=1,2, \ldots, \bullet \quad(11)
\end{gathered}
$$

где

○ $F o_{k}=\alpha_{k} F o, \alpha_{k}=b_{k} / a_{k}, \quad M_{k}=Q \alpha_{k}^{(1)} /\left(e b_{k}(k-1)\right) !$,

$B i_{k}=\left(G \cdot \alpha_{k}^{(1)}+S k \cdot\left(\alpha_{k}^{(4)}+4 S \alpha_{k}^{(3)}+6 S \alpha_{k}^{(2)}\right)\right) / b_{k}$.

Таким образом, исходная краевая задача (1) преобразована к линейной краевой задаче, относительно безразмерных величин, с граничными условиями 3-го рода.

Используя преобразование Лапласа, решение системы (11) для любого $k$ получим в виде [6]

$$
\theta^{(k)}=\frac{M_{k}}{B i_{k}}\left(1-\sum_{n=1}^{\infty} A_{k n} \cos \mu_{k n} X e^{-\mu_{k n}^{2} F o_{k}}\right)
$$

где

$$
A_{k n}=\frac{2 \sin \mu_{k n}}{\mu_{k n}+\sin \mu_{k n} \cos \mu_{k n}},
$$

a $\mu_{k n}-$ корни следующего характеристического уравнения:

$$
\mu_{k} \sin \mu_{k}-B i_{k} \cos \mu_{k}=0 .
$$

Суммируя (13) по $k$, согласно (5), окончательно получим решение поставленной краевой задачи

$$
\theta=\sum_{k=1}^{\infty} \frac{M_{k}}{B i_{k}}\left(1-\sum_{n=1}^{\infty} A_{k n} \cos \mu_{k n} X e^{-\mu_{k n}^{2} F o_{k}}\right),
$$

в котором $\theta_{P}$ определим из уравнения, полученного из (16) при $F_{k} \rightarrow \infty$, т. е.

$$
\theta_{P}=\sum_{k=1}^{\infty}\left(M_{k} / B i_{k}\right) .
$$

Определяя $\theta_{P}$ из уравнения (17), а по нему коэффициенты рядов Фурье (8), $M_{k}$ и $B i_{k}$ из (12), решение (16) будет полностью определено.

Частный случай полученного решения, в котором отсутствует высокотемпературный теплообмен с окружающей средой за счет излучения и не учитывается термочувствительность материала совпадает с точным решением [6].

По полученным результатам, числовые расчеты проделаны в среде MATLAB [12], с использованием алгоритма [4].

Результаты вычислений по определению температурных полей изотропных пластин, изготовленных из титанового сплава (на графике обозначается А), низкоуглеродистой стали (обозначается В) и алюминиевого сплава (обозначается С), проанализированы для следующих линейных аппроксимаций теплофизических характеристик рассмотренных материалов от температуры: 


$$
\begin{gathered}
\lambda(T)=\lambda_{0}+\delta_{\lambda} T=\lambda_{0}^{\prime}\left(1+\varepsilon_{\lambda} \theta\right) ; \quad \lambda_{0}^{\prime}=\lambda_{0}+\delta_{\lambda} T_{0} ; \\
\varepsilon_{\lambda}=\delta_{\lambda} T_{z} / \lambda_{0}^{\prime}, \\
C_{v}(T)=C_{v 0}+\delta_{c} T_{0}=C_{v 0}^{\prime}\left(1+\varepsilon_{c} \theta\right) ; C_{v 0}^{\prime}=C_{v 0}+\delta_{c} T_{0} ; \\
\varepsilon_{c}=\delta_{c} T_{z} / C_{v 0}^{\prime}, \\
\text { A. } \lambda_{0}=6,52 ; \quad \delta_{\lambda}=0,0172 ; \quad C_{v 0}=2079936 ; \\
\delta_{c}=1080 ; \quad \varepsilon=0,19, \\
\text { B. } \lambda_{0}=50,24 ; \quad \delta_{\lambda}=0,029 ; \quad C_{v 0}=3392840 ; \\
\qquad \begin{array}{r}
\delta_{c}=3883 ; \quad \lambda_{0}=143 ; \quad \delta_{\lambda}=0,0625 ; \quad C_{v 0}=2263250 ; \\
\delta_{c}=2082 ; \quad \varepsilon=0,057,
\end{array} \\
R=0,2, \alpha=30 ; 50 ; 70 ; 100, \quad T_{c}=420 ; 820 ; 1020 ; 1420 .
\end{gathered}
$$

На графиках введены такие обозначения для параметров и линий: $\theta \div T E T ; \alpha \div A F ; 1$ - пунктирная линия обозначает линейное решение; 2 штрихпунктирная, - нелинейность 1-го рода; 3 сплошная, - решение с двойной нелинейностью. Влияние нелинейностей анализируются на графиках по направлению снизу вверх.

На рис. 1 и 2 показаны распределения температурных полей в пластине, когда конвективный теплообмен превалирует над лучистым. Так как коэффициент теплопроводности алюминиевого сплава С имеет большое значение, то критерий Старка почти не оказывает влияния на нагрев пластины, поэтому линии 2 и 3 совпадают. При уменьшении $\lambda$ наблюдается вначале незначительное влияние нелинейности 1-го рода и двойной нелинейности на распределение температурного поля (сплав В). Дальнейшее уменьшение $\lambda$ приводит к качественному изменению расположения графиков, с (2-3-1) на (2-1-3), так как критерий Старка для титанового сплава уже имеет существенное значение $(S k=0,02)$, которое влияет на процесс теплообмена.

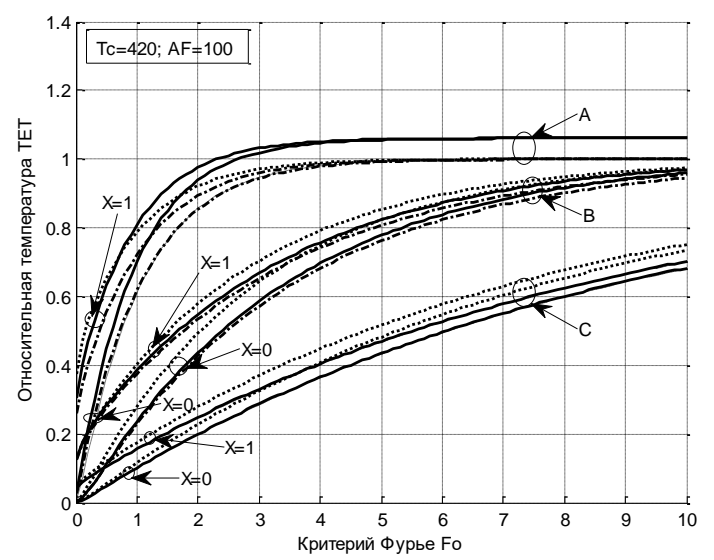

Рисунок 1 - Зависимость $\theta$ от критерия $F o$ при фиксированных значениях $T_{c}=420, \alpha=100$

На рис. 1 также видно, что с уменьшением коэффициента теплопроводности, при увеличении критерия Фурье, графики для всех случаев расчета сближаются, а для титанового сплава А, графики 1 и 2 , а также 3 совпадают для центра и поверхности пластины. Кроме того, разность между графиками 3 и совместно 1 и 2 практически не изменяется. На графике 2 для $F o=2$ проанализировано, как изменяется разность температур поверхности и центра пластины при уменьшении коэффициента теплопроводности, для случая двойной нелинейности (сплошные линии). Если спроектировать концы графиков 3 на ось относительной температуры, то можно установить, что при переходе от сплава С к сплаву В эта разность увеличивается, а затем, при переходе к сплаву А, существенно уменьшается.

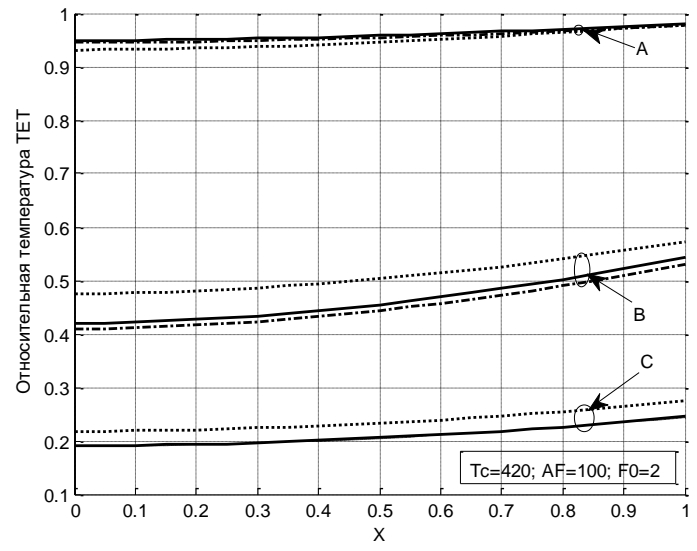

Рисунок 2 - Зависимость $\theta$ по толщине пластины при фиксированных значениях $T_{c}=420, \alpha=100$

Поскольку, начиная с $T_{c}=800^{\circ} \mathrm{C}$, теплообмен излучением является основным способом передачи тепла (около $85-95 \%$ тепла в печах передается на поверхность нагреваемых предметов излучением и лишь 5--15\% конвекцией), то на рис. 3 и 4 приведены расчеты для случая теплообмена $T_{c}=820, \alpha=70$.

На рис. 3 лучистый теплообмен уже превалирует над конвективным и в этом случае для сплава С, графики с нелинейностями 1-го рода и двойной нелинейностью уже не совпадают, особенно на поверхности пластины, так как критерий Старка $(S k=0,0023)$, и он уже оказывает влияние на процесс теплообмена. При уменьшении коэффициента теплопроводности и переходе к сплаву В, картина распределения графиков качественно меняется как на поверхности (2-1-3), так и в центре пластины (2-3-1). Дальнейшее уменьшение коэффициента теплопроводности и переход к сплаву А вызывает снова качественный переход в расположении графиков с (1-2-3) для центра на (2-1-3) для поверхности пластины, начиная с $F o=1$. При увеличении критерия Фурье, для этого сплава, начиная с $F o=4$, 5 , температурное поле распределяется только за счет лучистого теплообмена как на поверхности, так и в центре пластины. Наблюдается также в центре пластины для сплава В качественный переход распределения графиков, вначале (2-3-1) до критерия $F o=6$ а затем (2-1-3). 


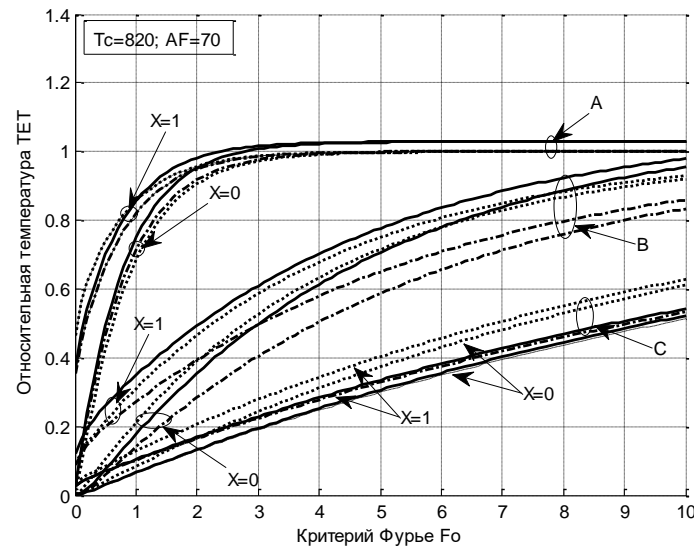

Рисунок 3 - Зависимость $\theta$ от критерия Fo при фиксированных значениях $T_{c}=820, \alpha=70$

На рис. 4, как и в предыдущем случае, разность температур будет существенной для сплава В, а затем она убывает и принимает незначительное значение для сплава А.

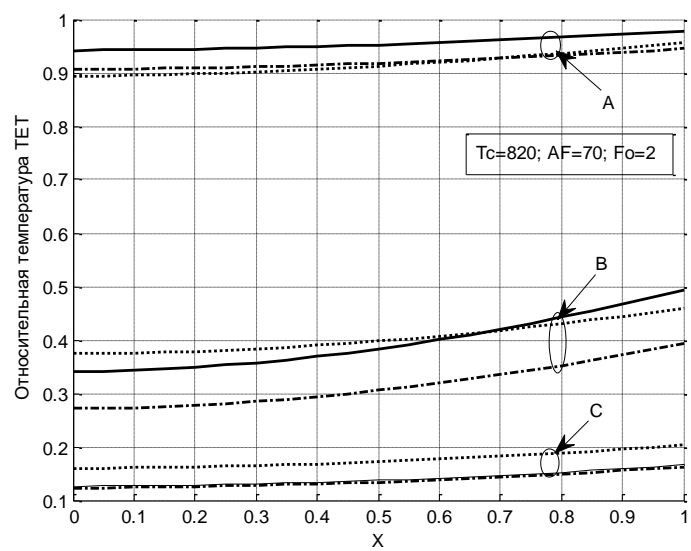

Рисунок 4 - Зависимость $\theta$ по толщине пластины при фиксированных значениях $T_{c}=820, \alpha=70$

На рис. 5 и 6 показаны распределения температур в пластине, когда лучистый теплообмен значительно превалирует над конвективным теплообменом $(S k=0,3)$. В этом случае, для сплава C, качественного изменения распределения графиков не происходит (2-3-1), как на поверхности, так и в центре пластины. Для сплава В, качественный переход распределения графиков с (2-3-1) на (2-1-3) происходит только до критерия Фурье $F o=1$, а затем сохраняется (2-1-3) как на поверхности, так и в центре пластины. При дальнейшем уменьшении коэффициента теплопроводности до сплава А, расхождения между графиками уменьшается, причем качественный переход происходит на поверхности пластины с (2-3-1) на (2-1-3), начиная с критерия Фурье $F o=$ 1. Для этого сплава, при увеличении критерия Фурье происходит сближение, а затем и совпадения графиков линейного решения и нелинейности первого рода как на поверхности, так и центре пластины. Такая же картина наблюдается и для случая двойной нелинейности. На рис. 6 видно, что разность между температурами поверхности и центра пластины будет существенной только для сплава В.

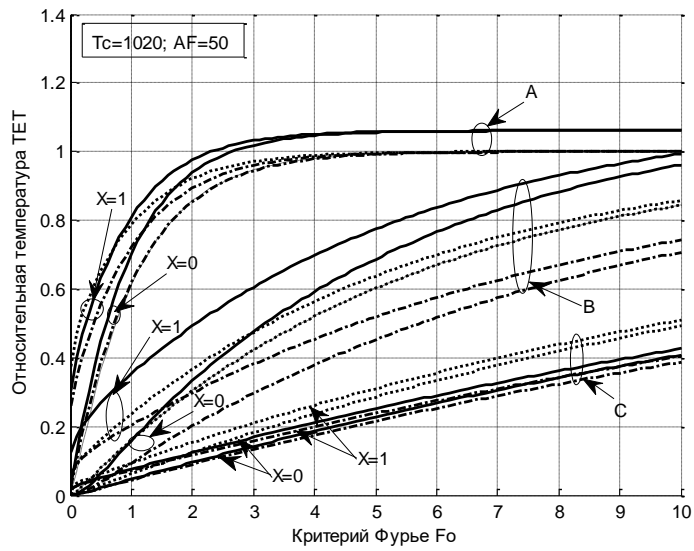

Рисунок 5 - Зависимость $\theta$ от критерия $F o$ при фиксированных значениях $T_{c}=1020, \alpha=50$

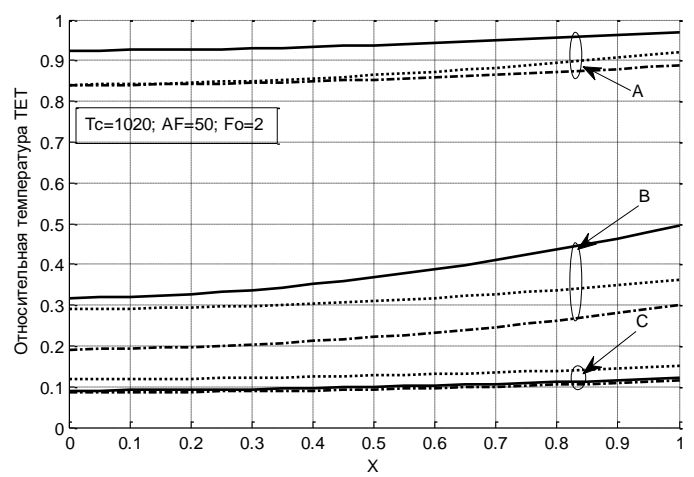

Рисунок 6 - Зависимость $\theta$ по толщине пластины при фиксированных значениях $T_{c}=1020, \alpha=50$

На рис. 7, для сплавов А и В, показано влияние нелинейностей в зависимости от критерия Фурье при значении критерия Старка $S k=0,5$, что соответствует температуре среды $T_{c}=1420^{\circ} \mathrm{C}$. В сплаве В качественного перехода не происходит как на поверхности, так и центре пластины начиная с критерия Фурье $F o=2$. Для сплава А также происходит сближение графиков по линейным решениям и нелинейностям 1-го рода, которые, начиная с критерия Фурье $F o=8$, совпадают. Решения по двойным нелинейностям как на поверхности, так и центре пластины совпадают начиная с критерия $F o=4$.

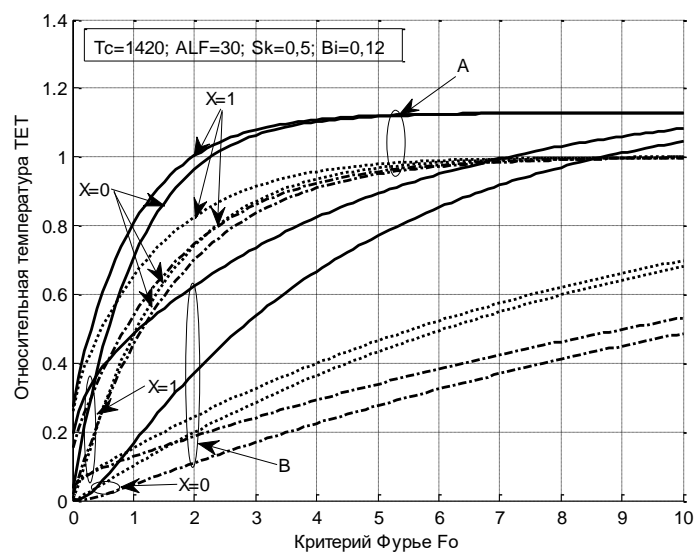

Рисунок 7 - Зависимость $\theta$ от критерия $F o$ при фиксированных значениях $T_{c}=1420, \alpha=30$ 
ВЫВОДЫ. 1. При нагревании пластины, когда конвективный теплообмен превалирует над лучистым, коэффициент теплопроводности алюминиевого сплава имеет большое значение и критерий Старка почти не оказывает влияния на нагрев пластины. При уменьшении коэффициент теплопроводности до титанового сплава А, происходит качественное изменение расположения графиков, так как критерий Старка уже имеет существенное значение, которое значительно влияет на процесс теплообмена.

2. При увеличении температуры среды и превалировании лучистого теплообмена над конвективным, для алюминиевого сплава графики с нелинейностями 1-го рода и двойной нелинейностью не совпадают, особенно на поверхности пластины, так как критерий Старка $(S k=0,0023)$ уже оказывает влияние на процесс теплообмена. При уменьшении коэффициента теплопроводности и переходе к низкоуглеродистой стали, картина распределения графиков качественно меняется как на поверхности $(2-1-3)$, так и в центре пластины $(2-3-1)$. Дальнейшее уменьшение коэффициента теплопроводности и переход к титановому сплаву вызывает снова качественный переход в расположении графиков с $(1-2-3)$ для центра на $(2-1-3)$ для поверхности пластины, начиная с критерия Фурье $F o=1$.

3. При существенном превалировании лучистого теплообмена над конвективным $(S k=0,3)$, для алюминиевого сплава качественного изменения распределения графиков не происходит $(2-3-1)$ как на поверхности, так и в центре пластины. Для низкоуглеродистой стали, качественный переход распределения графиков с $(2-3-1)$ на $(2-1-3)$ происходит только до критерия Фурье $F o=1$, а затем сохраняется $(2-1-3)$ как на поверхности, так и в центре пластины. При дальнейшем уменьшении коэффициента теплопроводности до титанового сплава, расхождения между графиками уменьшается, причем качественный переход происходит на поверхности пластины с $(2-3-1)$ на $(2-1-3)$, начиная с критерия Фурье $F o=1$. Для этого сплава, при увеличении критерия Фурье происходит сближение, а затем и совпадения графиков линейного решения и нелинейности первого рода как на поверхности, так и центре пластины. Такая же картина наблюдается и для случая двойной нелинейности.

Дальнейшие исследования будут направлены на сравнения влияния нелинейностей и анизотропии для ортотропной пластины в широком диапазоне изменения коэффициента теплопроводности.

\section{ЛИТЕРАТУРА}

1. Зайцев Е. П. Суперпозиция основных уравнений квазистатической термоупругости анизотропных термочувствительных тел. Вісник Кременчуцького національного університету імені Михайла Остроградського. 2017. Вип. 1 (102) С. 22-31.

2. Белецкий В. М. Алюминиевые сплавы: справочник. К.: Коминтех, 2005. 365 с.

3. Зайцев Е. П. Влияние термочувствительности и зонального высокотемпературного теплообмена на температурное поле бесконечного цилиндра. Вісник Кременчуцького національного університету імені Михайла Остроградського. 2016. Вип. 3 (98), част. 1. С. 33-40.

4. Кудинов В. А., Аверин Б. В., Стефанюк Е. В. Анализ нелинейного теплопереноса на основе определения фронта температурного возмущения. Tenлофизика высоких температур. 2005. № 4. С. 1-9.

5. Карташов Э. М. Аналитические методы в теории теплопроводности твердых тел. М: Высшая школа, 2001. $550 \mathrm{c}$.

6. Кузнецова Е. Л. Моделирование теплопереноса в нелинейном анизотропном пространстве на основе аналитического решения. Математическое моделирование. 2012. Т. 24, № 1. С. 46-54.

7. Формалев В. Ф. Теплопроводность анизотропных тел. Аналитические методы решения задач. М.: Физматлит, 2015. 312 с.

8. Процюк Борис, Горун Олег. Вплив конвективно-променевого теплообміну на температурне поле півбезмежного термочутливого трискладового тіла за дії джерела тепла. Математические проблемы механики неоднородных структур / под общ. ред. И. А. Луковского, Г. С. Кита, Р. М. Кушнира. Львов: Институт прикладных проблем механики и математики им. Я. С. Подстригача НАН Украины. 2014. C. $148-150$.

9. Noda N. Thermal stresses in materials with temperature-dependent properties. Thermal Stresses I. Amsterdam: Elsevier, 1986. P. 391-483.

10. Ozisik M. Necati Heat conduction. Department of Mechanical and aerospace engineer. North Carolina State University, Raleigh. New York: 1980. 687 p.

11. Тихонов А. Н., Самарский А. А. Уравнения математической физики. М.: Изд. МГУ, 1999. 799 с.

12. Кетков Ю. Л., Кетков А. Ю., Шульц М. М. MATLAB 7: программирование, численные методы. СПб.: БХВ Петербург, 2005. 752 с.

\section{COMPARISON OF INFLUENCE OF NONLINEARITIES OF THE FIRST SORT AND DOUBLE NONLINEARITY ON HEAT CONDUCTIVITY OF PLATES OF ALUMINIUM, TITANIUM AND STEEL ALLOYS}

\section{Y. Zaytsev}

Flight Academy of National Aviation University

vul. Dobrovolsky, 1, Kropyvnytsky, 25005, Ukraine. E-mail: zaytsev.ep.klanau@ gmail.com

Purpose. The research objective is the application of a new approach to solution of problems of heat conductivity for impact assessment of nonlinearities of the first sort and double nonlinearity on distribution of temperature fields in aluminum and titanium alloys which have essential coefficients distinctions of heat conductivity. At the present stage of development of technology, it is necessary to provide as much as possible strength, reliability and durability of the structural materials which successfully function in the conditions of high-temperature heat exchange. In this regard, a 
broad application is found by the recently received new alloys of aluminum and titanium used for the instruments of space vehicles and aircraft, steam and gas turbines, jet and rocket engines. Methodology. Initially to the original problem, transformed to the dimensionless form, Goodman's and Kirchhoff's transformation should be applied, then, the relative temperature and its functions are expanded according the sines on the priori interval. Further on, the principle of superposition is applied, and the initial setting is transformed in totality of linearized problems. Linear problems can be solved by integral transformation method, and then they are summarized. The upper boundary of priori interval is defined according to the condition that the relative temperature, derived from the problem solution, at the problem values $\rho, \eta$ and $F 0 \rightarrow \infty$, possesses the value of the priori interval upper boundary. Findings. The solution problems method of nonlinear heat conductivity and quasistatic thermoelasticity is developed and approved. Originality. The development of an approximate analytical method and receiving qualitative and quantitative numerical results for impact assessment of nonlinearities on distribution of the temperature fields in structural elements made from aluminum and titanium alloys, allows considering the properties of the material and high-temperature heat exchange with the environment. Practical value. The received results can be used in estimated practice of heat-technical laboratories for the assessment of the experimental material studies while being subjected to the impact of high-temperature heat exchange, and they may also serve as a criterion for the evaluation of the appropriate numerical solutions. Conclusions. Further researches will be directed to comparisons of influence of nonlinearities and anisotropy for an orthotropic plate with a broad range of the coefficient change of heat conductivity.

Key words: heat (thermal) conductivity, superimposition, thermal sensitivity, radiant-convective heat exchange, Goodman's and Kirchhoff's transformations, Fourier series.

\section{REFERENCES}

1. Zaytsev, Y. P. (2017), "Superposition of the basic equations of quasi-static thermoelasticity of anisotropic thermosensitive bodies", Transactions of Kremenchuk Mykhailo Ostrohradskyi National University, Vol. 1 (102), pp. $22-31$.

2. Beletsky, V. M. (2005), Aluminum alloys: Reference, $\mathrm{K}$.: Comintech.

3. Tikhonov, A. N., Samarskyy, A. A. (1999), "Uravneniya matematicheskoy fiziki [ Equations of mathematical physics]", Publ. house MGU, Moscow, Russia.

4. Zaytsev, Y. P. (2016), "Influence of the double nonlinearity on the infinite cylinder temperature field at zonal high-temperature heat exchange", Transactions of Kremenchuk Mykhailo Ostrohradskyi National University, Vol. 3 (98), part 1, pp. $33-40$.

5. Kudinov, V. A., Averin, B. V., Stephanyuk, E. V. (2005), "Nonlinear heat transfer analysis conditioned upon the temperature perturbation edge detection", High temperatures thermal physics, Vol. 4, pp. 1-9.

6. Kartashov, E. M. (2012), "Analiticheskie metody $v$ teorii teploprovodnosti tverdykh tel [Analytical methods in solid bodies thermal conduction theory]", Higher school, Moscow, Russia.
7. Kuznetsova, E. L. (2012), "Simulation of heat transfer in nonlinear anisotropic space based on the analytical solution”, Mathematical modeling, Vol. 24, № 1, pp. 46-54.

8. Formalev, V. F. (2015), “Teploprovodnost anizotropnykh tel. Analiticheskie metody resheniya zadach [Anisotropic bodies heat conductivity. Analytical methods for problems solutions]", Fizmatlit, Moscow, Russia.

9. Protsyuk, V., Horun, O. (2014), "The influence of a convective-radiant heat exchange on the temperature field of half-infinite thermosensitive three-layer body under the action of a heat source", Mathematical Problems of Mechanics of Nonhomogeneous Structures, Pidstryhach Institute for Applied Problems of Mechanics and Mathematics NAS, pp. 148-150.

10. Noda, N. (1986), "Thermal stresses in materials with temperature-dependent properties", In: Thermal Stresses I. Amsterdam: Elsevier, pp. 391 - 483.

11. Ozisik, M., Necati, (1980), "Heat conduction", Department of Mechanical and aerospace engineer, North Carolina State University, Raleigh. New York, $687 \mathrm{p}$.

12. Ketkov, Y. L, Ketkov, A. Y, Shults, M. M. (2005), MATLAB 7: programmirovanie, chislennue metodu[ MATLAB 7: programming, numerical methods ], Science, Sankt-Petersburg, Russia. 